\title{
Instabilities of thermocapillary flows between counter-rotating disks under microgravity conditions
}

\author{
Qi-Sheng Chen ${ }^{\mathrm{a}, \mathrm{b}, *}$, Meng He ${ }^{\mathrm{b}}$, Peng Zhu ${ }^{\mathrm{b}}$, Kai-Xin $\mathrm{Hu}^{\mathrm{a}, 1}$ \\ ${ }^{a}$ Key Laboratory of Microgravity, Institute of Mechanics, Chinese Academy of Sciences, Beijing 100190, China \\ ${ }^{\mathrm{b}}$ School of Engineering Science, University of Chinese Academy of Sciences, Beijing 100190, China
}

\section{A R T I C L E I N F O}

\section{Article history:}

Received 31 March 2017

Received in revised form 9 August 2017

Accepted 30 September 2017

Available online 12 October 2017

\begin{abstract}
A B S T R A C T
Instabilities of thermocapillary flows between counter-rotating disks under microgravity conditions are investigated by linear stability analysis. The basic-state and perturbation equations are solved using the Chebyshev-collocation method. For small Prandtl number liquids $(\operatorname{Pr} \leq 0.01)$, bifurcation of thermocapillary flows between counter-rotating disks is found to be a 3D oscillatory state for the Coriolis number $\tau \leq$ 100 , except at certain Coriolis number where the most unstable perturbation is 3D stationary state. The critical capillary Reynolds number is a function of Prandtl number, Coriolis number and aspect ratio.

Energy analysis shows that the perturbation energy consists of the viscous dissipation, the work done by surface tension and the interaction between the perturbation flow and the basic flow, respectively. For small Prandtl number liquids ( $P r \leq 0.01$ ), the perturbation energy mainly comes from the interaction between the perturbation and the basic flow, which suggests that the instability mechanism is hydrodynamic. The interaction between the perturbation and the basic flow in the azimuthal direction becomes negative when a moderate rotation is applied on the disks, and the moderate rotation can stabilize the thermocapillary flows for small Prandtl number liquids.
\end{abstract}

(c) 2017 Elsevier Ltd. All rights reserved.

\section{Introduction}

Thermocapillary flows are important in the floating-zone, Czochralski crystal growth [1] and microgravity materials processing [2]. The phenomena arisen from thermocapillary flows could interact with other transport and phase-change phenomena. Campbell et al. [3] studied the macrosegregation in the crystals grown with the floating-zone technique. A germanium seed with a diameter of $8 \mathrm{~mm}$ was installed inside an ampoule. When seed rotation changed from 0 to $2 \mathrm{rpm}$, a striation pattern appeared in the crystal. In this growth process, there exist melting and solidifying interfaces at the top and bottom of melt. To investigate the mechanism of the oscillatory convection which may cause striations in the crystals grown by the floating-zone technique, liquid bridge is usually used to imitate half of the floating zone [1]. Many researchers performed numerical simulations on the thermocapillary flows in liquid bridges. For crystal-growth applications, the melts are usually liquids with $\operatorname{Pr} \ll 1$. The Prandtl numbers for germanium, silicon, gallium arsenide are $0.008,0.027$,

\footnotetext{
* Corresponding author at: Key Laboratory of Microgravity, Institute of Mechanics, Chinese Academy of Sciences, Beijing 100190, China.

E-mail address: qschen@imech.ac.cn (Q.-S. Chen).

1 Current address: School of Mechanical Engineering and Mechanics, Ningbo University, Ningbo 315211, China.
}

0.068 , respectively [4]. For small Prandtl numbers $(\operatorname{Pr} \ll 1)$ the first instability of the axisymmetric flow is a stationary bifurcation which is considered hydrodynamic in nature [5-9]. For liquid bridges with Prandtl numbers $\operatorname{Pr} \geq 1$, the first instability is oscillatory which is analogous to the hydrothermal-wave instability [5,10-12]. There are two different oscillatory instabilities for high Prandtl number silicone oils $(\mathrm{Pr}=30$ and 74$)$ : a pulsating standing wave and a rotating traveling wave [13]. In the experiments, the onset oscillation can be either a traveling wave or a pulsating standing wave, depending on the perturbation introduced at the beginning of the experiments. The pulsating standing wave results from the superposition of two counter-propagating azimuthal hydrothermal waves with equal amplitude.

In production of crystals by the floating zone method, the feed and crystal rods are often rotating in order to suppress the azimuthal asymmetry. A model of liquid bridge with counterrotating disks can be proposed to study stability phenomena in a half part of the zones (Fig. 1). Here, the liquid bridge is formed between two disks of diameter $2 R_{0}$, which are separated by a distance $L$. Two temperatures $T_{\mathrm{L}}$ and $T_{\mathrm{L}}+\Delta T$ are applied to the lower and upper disks. We assume that the two coaxial disks are counterrotating with the same angular velocity.

The purpose of this paper is to study instability mechanism of the thermocapillary flows between counter-rotating disks by linear stability analysis. The impacts of Prandtl number, the aspect ratio 


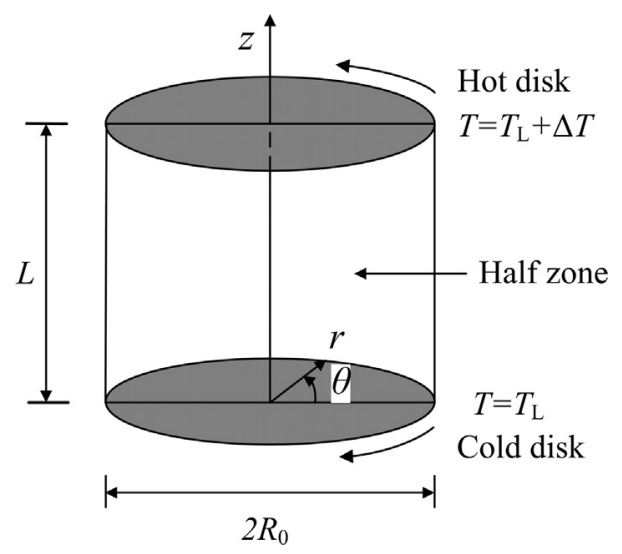

Fig. 1. Schematic of a liquid bridge between counter-rotating disks.

and the Coriolis number on the critical capillary Reynolds number are investigated. In Section 2, the physical and mathematical models and numerical methods are described. In Section 3, we calculate the critical capillary Reynolds numbers and critical frequencies for different Coriolis numbers.

\section{Problem formulation}

Typical dimensionless parameters such as aspect ratio, Marangoni number, Prandtl number, capillary Reynolds number and Coriolis number are defined as,

$A=\frac{L}{2 R_{0}}, \quad M a=\frac{U_{0} R_{0}}{\alpha}, \quad \operatorname{Pr}=\frac{v}{\alpha}, \quad R e_{\gamma}=\frac{M a}{\operatorname{Pr}}, \quad \tau=\Omega R_{0}^{2} / \nu$,

where the reference velocity is $U_{0}=\left|\sigma_{T}^{\prime}\right| \Delta T / \rho_{0} v$, and $\rho_{0}, v, \alpha, \sigma_{T}^{\prime}$ denote liquid density, kinematic viscosity, thermal diffusivity, surface tension derivative with respect to temperature, respectively. $\Omega$ is the angular velocity of the upper disk, which is positive when rotating counter-clockwise viewing from the top.

The dimensionless governing equations and boundary conditions for the basic state fields can be obtained as in Ref. [14]. In linear stability analysis of thermocapillary convections, the small amplitude fluctuations of the velocities $\mathbf{u}=(u, v, w)$, pressure $p$ and temperature $T$ are imposed on the basic state. The dimensionless governing equations for the perturbation quantities can be obtained as $[11,14]$,

$\nabla \cdot \mathbf{u}=0$

$\frac{\partial \mathbf{u}}{\partial t}+\mathbf{u} \cdot \nabla \mathbf{u}_{0}+\mathbf{u}_{0} \cdot \nabla \mathbf{u}=-\nabla p+\nabla^{2} \mathbf{u}$

$\frac{\partial T}{\partial t}+\mathbf{u} \cdot \nabla T_{0}+\mathbf{u}_{0} \cdot \nabla T=\frac{1}{P r} \nabla^{2} T$.

After applying the curl operator to the momentum equation (2b) to eliminate the pressure term, we can obtain the vorticity equations. For solving the eigenvalue problem, the continuity equation (2a), the vorticity equations in $r$ and $z$ directions, and the energy equation (2c) are used for $m>0$, while the continuity equation, the vorticity equation in the azimuthal direction, and the energy equation are used for $m=0$. Here, $m$ denotes the azimuthal wavenumber of perturbation. The continuity equation is included in the perturbation equations to ensure that the continuity equation is satisfied at all grid points. The boundary conditions for the perturbation equations are set as in the following.

The boundary conditions at the disks $(z=0$ and $z=2 A)$ are given by,

$\mathbf{u}=0$ and $T=0$.
For $m=0$, the continuity equations $\frac{\partial w}{\partial z}=0$ at $z=0$ and $z=2 A$ are included in the perturbation equations, and the vorticity equations in the azimuthal direction at grid points adjacent to the solid disks are not included. The boundary conditions at the free surface are,

$\mathbf{u} \cdot \mathbf{n}=0, \quad \mathbf{t} \cdot \mathbf{S} \cdot \mathbf{n}=-\frac{M a}{P r} \mathbf{t} \cdot \nabla T$,

$\mathbf{s} \cdot \mathbf{S} \cdot \mathbf{n}=-\frac{M a}{P r} \mathbf{s} \cdot \nabla T, \quad$ and $\mathbf{n} \cdot \nabla T=0$,

where $\mathbf{s}$ denotes the tangential unit vectors in the horizontal crosssections. The conditions at the central axis, $r=0$, are taken as [11],

$u=0, \quad \frac{\partial w}{\partial r}=0, \quad \frac{\partial T}{\partial r}=0, \quad$ for $\quad m=0$,

$\frac{\partial u}{\partial r}=0, \quad u+\frac{\partial v}{\partial \theta}=0, \quad w=0, \quad T=0, \quad$ for $\quad m=1$,

$u=0, \quad v=0, \quad w=0, \quad T=0, \quad$ for $\quad m>1$.

The perturbation quantities $(u, v, w, p, T)$ can be expanded as a sum of normal modes,

$\left(\begin{array}{c}u \\ v \\ w \\ p \\ T\end{array}\right)=\sum_{m} e^{\sigma t+i m \theta}\left(\begin{array}{c}\tilde{u}_{m}(r, z) \\ i m \tilde{v}_{\mathrm{m}}(r, z) \\ \tilde{w}_{m}(r, z) \\ \tilde{p}_{m}(r, z) \\ \tilde{T}_{m}(r, z)\end{array}\right)+$ c.c.

where $\sigma=\sigma_{r}+i \sigma_{i}, \sigma_{r}$ and $\sigma_{i}$ are the growth rate and frequency of small perturbation, respectively, $i$ denotes the complex unit $\sqrt{-1}$, and c.c. denotes the complex conjugate.

The basic state and perturbation equations are solved using the Chebyshev-collocation method. For calculation of the basic-state flow and temperature fields, the numerical computations are based on a pseudo-spectral Chebyshev method [15]. For cases with large capillary Reynolds numbers, we use $49 \times 129$ Chebyshev polynomials in $r$ and $z$ directions. The dimensionless time step for the basic state calculation is set as $\Delta t=10^{-7}$. For Calculation of the perturbations, $37 \times 97$ Chebyshev polynomials are used. The eigenvalues and eigenfunctions are then obtained using the $\mathrm{Q}-\mathrm{R}$ method. Since four perturbation equations are used, the complex matrix for the generalized eigenvalue problem has a size of (4MN) [2] with $M=37$ and $N=97$. Linear interpolations for both $\operatorname{Re}_{\gamma_{\mathrm{c}}}$ and $\sigma_{i_{c}}$ are adopted

\section{Results}

\subsection{Dependence of the critical capillary Reynolds number on the aspect ratio}

Fig. 2a and b shows dependence of the critical capillary Reynolds number $R e_{\gamma_{c}}$ on the aspect ratio $A$ for liquid bridges with $P r=0.01$ and $P r=0.001$, respectively. The most unstable modes are indicated near symbols in the figures, except those which have the mode $m=1$. There are still some differences of features between the modes for different Coriolis numbers. When no rotation is applied, the region of the mode $m=2$ is $A=0.5-0.8$ for both $\operatorname{Pr}=0.01$ and 0.001 . When the Coriolis number $\tau=50$, the region of the mode $m=2$ is only at $A=0.5$ for both $P r=0.01$ and 0.001 . When $\tau=100$, the region of the mode $m=2$ ranges from $A=0.8$ to 1.05 for both $\operatorname{Pr}=0.01$ and 0.001 .

The critical capillary Reynolds numbers for $\mathrm{Pr}=0.001$ are generally smaller than those for $P r=0.01$, and the differences are about $4.9-9.7 \%$ of the values for $P r=0.01$. Instabilities are caused by the interaction between the perturbation and the basic flow for $\operatorname{Pr} \leq$ 0.01 . However, the dissipating effect of the thermocapillary force 


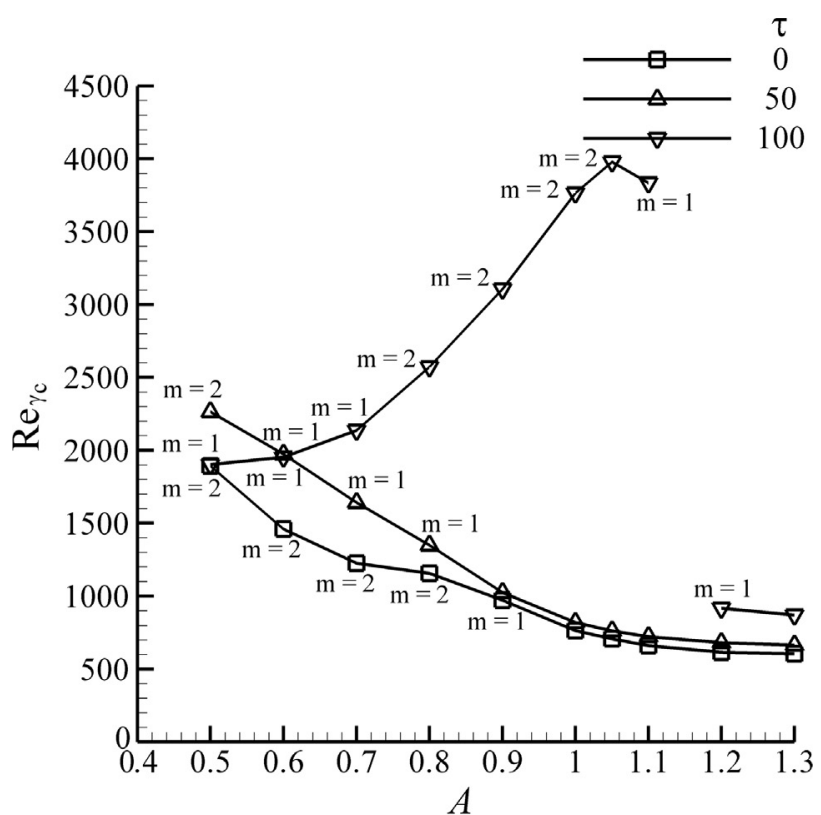

(a)

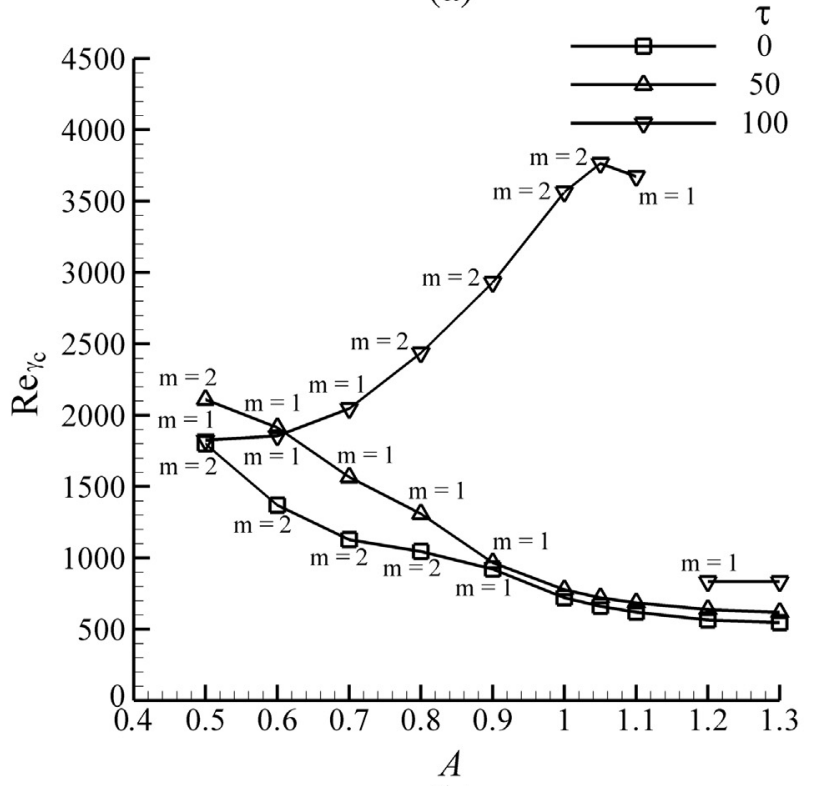

(b)

Fig. 2. Dependence of the critical capillary Reynolds number on the aspect ratio for (a) $\operatorname{Pr}=0.01$ and (b) $\operatorname{Pr}=0.001$

can be seen for $\operatorname{Pr}=0.01$ which is responsible for the higher critical values, while the dissipating effect of the thermocapillary force is negligible for $\operatorname{Pr}=0.001$. When the Coriolis numbers is small $(\tau=50)$, the critical capillary Reynolds number $R e_{\gamma_{c}}$ decreases with the aspect ratio. When the Coriolis number is large $(\tau=100)$, the curve of the critical capillary Reynolds number vs aspect ratio has two branches, and there is a maximum of the critical capillary Reynolds number $\operatorname{Re}_{\gamma_{c}}$ at around $A=1.05$.

The critical frequencies $\sigma_{i_{c}}$ for the most unstable mode vs the aspect ratio are plotted in Fig. $3 a$ and $b$ for $\operatorname{Pr}=0.01$ and 0.001 , respectively. The critical frequencies are zero for the static liquid bridges. When the aspect ratio increases for $\tau=50$, the critical frequency changes from positive to negative, and there exists a stationary bifurcation around the aspect ratio $A=0.85$. The curve of the critical frequency vs aspect ratio has two branches for $\tau=100$.

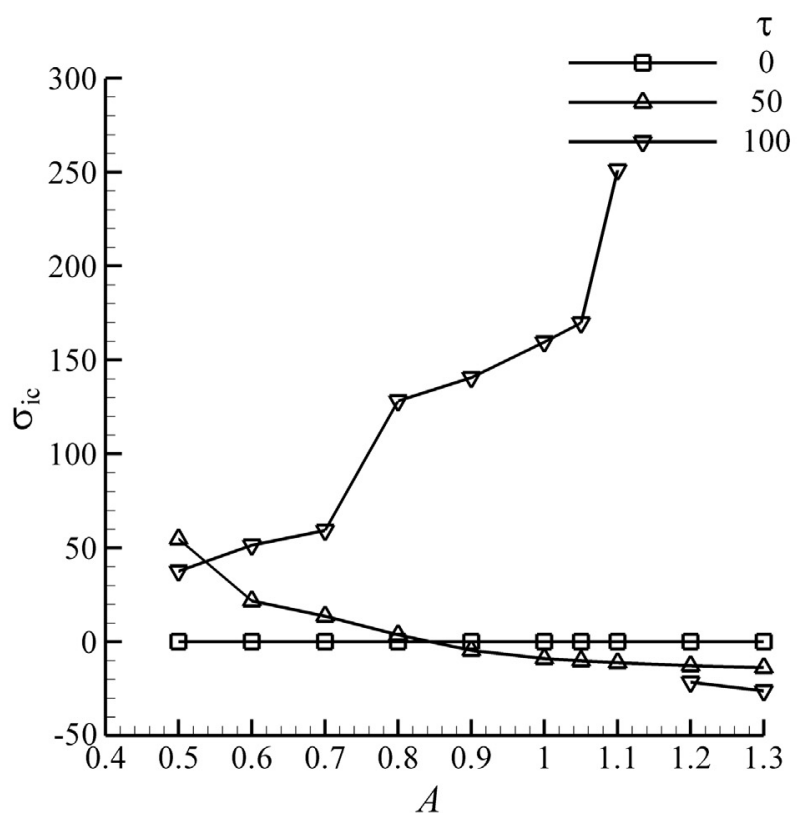

(a)

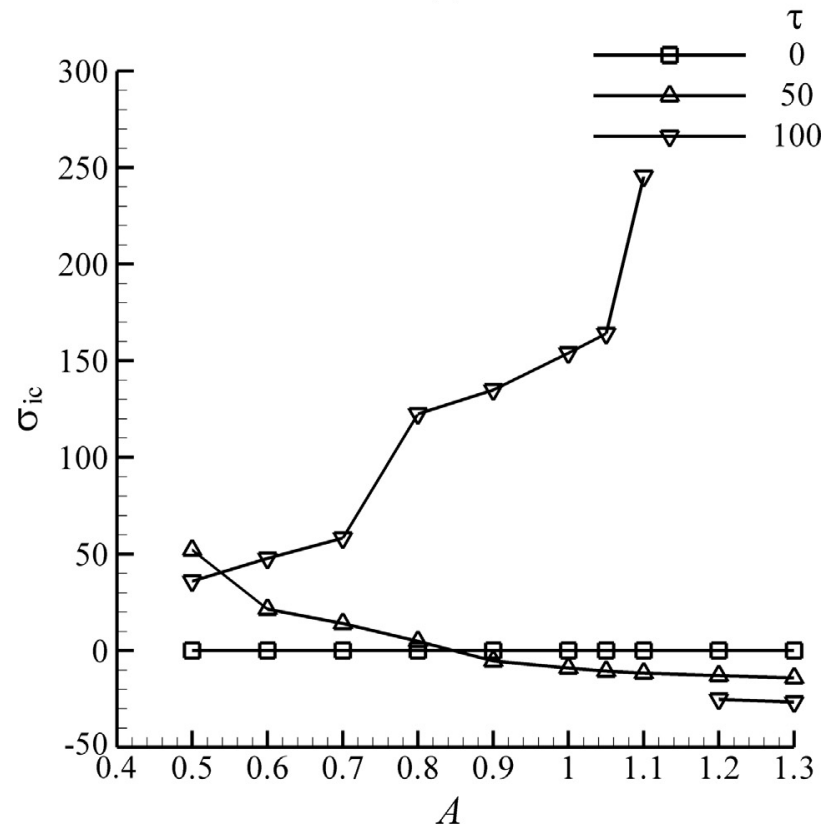

(b)

Fig. 3. Dependence of the critical frequency on the aspect ratio for (a) $\operatorname{Pr}=0.01$ and (b) $\operatorname{Pr}=0.001$.

Generally, the critical capillary Reynolds numbers for bridges with large aspect ratios are lower than those for bridges with small aspect ratios. This is conceivable as the increasing of the distance between two disks can weaken the constraint of flows. However, anomalies appear when strong rotations are applied. When $\tau \leq$ 50 , our computations show that only one vortex exists when the aspect ratio is in the range of $A=0.5-1.3$. However, the basic flow field for $\tau=100$ changes dramatically with different aspect ratio. For the aspect ratio in the range of 0.5-1.1, the basic flow structure has two vortexes (Fig. 4a and b) while there is only one vortex in the basic flow structure for $A=1.2$ and 1.3. The appearance of the secondary vortex at high Coriolis numbers can stabilize the basic flow significantly. So the increasing of distance between two disks changes the flow structure and leads to the anomalous 


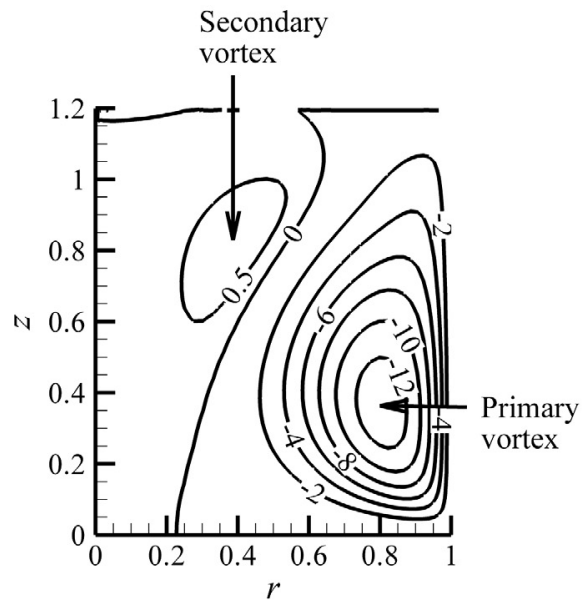

(a)

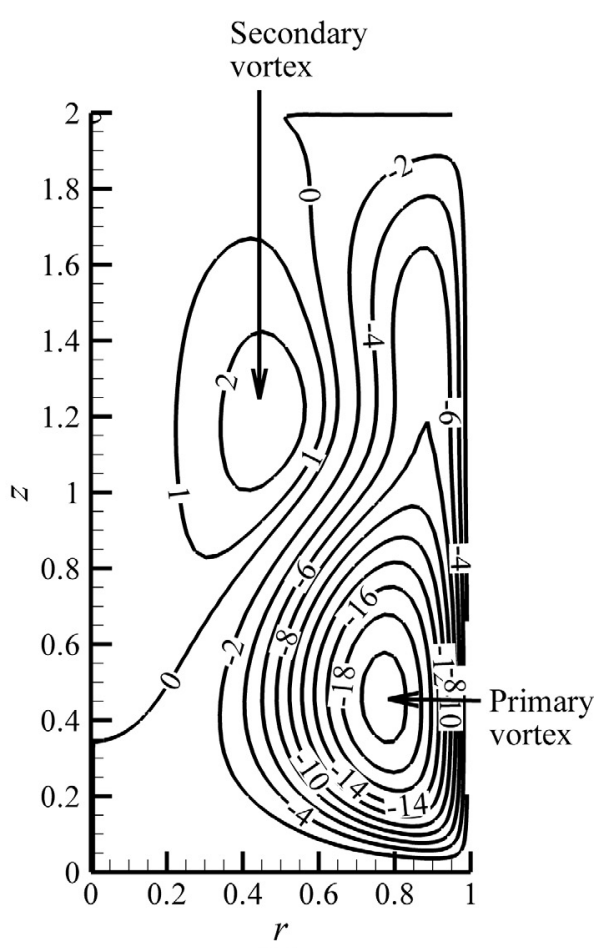

(b)

Fig. 4. (a) Contours of streamfunction in liquid bridges for $A=0.6$ and $R e_{\gamma}=2000$, (b) Contours of streamfunction for $A=1$ and $R e_{\gamma}=3800$. Other parameters are $P r=0.01$ and $\tau=100$.

dependence of the critical capillary Reynolds number on the aspect ratio.

Now we summarize dependence of the critical capillary Reynolds number and frequency on rotation. For small Prandtl number liquids $(\operatorname{Pr} \leq 0.01)$, the most unstable mode between static disks is a stationary secondary flow $\left(\sigma_{i}=0\right)$, and the bifurcation is from the axisymmetric steady state to a non-axisymmetric steady state. The instabilities are considered purely hydrodynamic and the perturbation of the temperature on the free surface is not important for the instability mechanism $[7,8]$. The bifurcation of thermocapillary flows between counter-rotating disks is from an axisymmetric state to an oscillatory one except at certain Coriolis number where the most unstable perturbation is 3D stationary. The most unstable mode is usually a traveling hydrothermal wave. The critical frequency changes with the Coriolis number significantly, and the direction of wave propagation also changes at higher Coriolis numbers.

\subsection{Energy analysis}

In this section, onset of the most unstable mode is studied by the energy analysis. Changing rate of the perturbation energy can be obtained as follows [7], $\frac{\partial E_{k i n}}{\partial t}=\frac{1}{2} \frac{\partial}{\partial t} \int \mathbf{u}^{2} d^{3} r=-D+M_{z}+M_{\theta}+I_{u 0}+I_{w 0}+I_{v 0}$.

Here, the viscous dissipation $D=\frac{1}{2} \int(\mathbf{S}: \mathbf{S}) d^{3} r$, the strain-rate tensor $\mathbf{S}=\nabla \mathbf{u}+\mathbf{u} \nabla$, the interaction between the perturbation flow and the basic flow. $M_{z}=\int w\left(\frac{\partial u}{\partial z}+\frac{\partial w}{\partial r}\right) r d z d \theta$ and $M_{\theta}=\int v\left(\frac{\partial v}{\partial r}+\frac{1}{r} \frac{\partial u}{\partial \theta}-\frac{v}{r}\right) r d z d \theta$ are the work done by surface tension in the axial and azimuthal directions, respectively. $I_{u 0}=-\int\left(u_{0} \frac{v^{2}}{r}+u^{2} \frac{\partial u_{0}}{\partial r}+u w \frac{\partial u_{0}}{\partial z}\right) d^{3} r, \quad I_{w 0}=-\int\left(u w \frac{\partial w_{0}}{\partial r}+w^{2} \frac{\partial w_{0}}{\partial z}\right) d^{3} r$, and $I_{v 0}=-\int\left(-v_{0} \frac{u v}{r}+u v \frac{\partial v_{0}}{\partial r}+v w \frac{\partial v_{0}}{\partial z}\right) d^{3} r$ are the interactions between the perturbation flow and the basic flows in radial, axial and azimuthal directions, respectively.

In Table 1, changing rates of the perturbation energy for different Prandtl numbers are compared. It can be found that, for small Prandtl number liquids $(\operatorname{Pr} \leq 0.01)$, the perturbation energy mainly comes from the interaction between the perturbation flow and the basic flow. It suggests that although the perturbation is usually a traveling wave when the disks are rotating, the instability is hydrodynamic in nature. The interaction between the perturbation and the basic flow in the azimuthal direction, $I_{v 0}$, becomes negative when the Coriolis number is 50 (Table $1 \mathrm{~b}$ ), which means a moderate rotation can stabilize thermocapillary flows for small Prandtl

Table 1

Changing rates of the perturbation energy for different Prandtl numbers at three Coriolis numbers.

\begin{tabular}{|c|c|c|c|c|c|c|c|c|}
\hline $\operatorname{Pr}$ & $R e_{\gamma}$ & $\sigma_{r}+i \sigma_{i}$ & $m$ & $M_{z} / D$ & $M_{\theta} / D$ & $I_{u 0} / D$ & $I_{w 0} / D$ & $I_{v 0} / D$ \\
\hline \multicolumn{9}{|c|}{ (a) $A=0.5, \tau=0$} \\
\hline 0.001 & 2000 & $5.14+0 \mathrm{i}$ & 2 & 0. & -0.001 & -0.091 & 1.149 & 0 \\
\hline 0.01 & 2000 & $2.54+0 \mathrm{i}$ & 2 & -0.003 & -0.008 & -0.093 & 1.135 & 0 \\
\hline \multicolumn{9}{|c|}{ (b) $A=0.5, \tau=50$} \\
\hline 0.001 & 2500 & $9.04+54.7 i$ & 2 & 0 & -0.001 & -0.081 & 1.233 & -0.047 \\
\hline 0.01 & 2500 & $4.98+57.1 \mathrm{i}$ & 2 & -0.003 & -0.008 & -0.083 & 1.203 & -0.046 \\
\hline \multicolumn{9}{|c|}{ (c) $A=0.5, \tau=100$} \\
\hline 0.001 & 2000 & $3.34+38.9 i$ & 1 & 0. & 0. & 0.099 & 0.527 & 0.421 \\
\hline 0.01 & 2000 & $1.71+38.9 \mathrm{i}$ & 1 & 0.001 & -0.001 & 0.100 & 0.507 & 0.417 \\
\hline
\end{tabular}


number liquids. However, a strong rotation can destabilize the thermocapillary flows (Table $1 \mathrm{c}$ ).

\section{Conclusions}

Linear stability analysis of thermocapillary flows between counter-rotating disks with Prandtl numbers $\operatorname{Pr} \leq 0.01$ was performed. The Coriolis numbers for the current calculations are in the range of $0-100$. The basic flow and temperature solutions are obtained by using the pseudo-spectral Chebyshev method. The perturbation equations are discretized with Chebyshev polynomial expansions in the radial and vertical directions.

Energy analysis shows that the perturbation energy consists of the viscous dissipation, the work done by surface tension and the interaction between the perturbation flow and the basic flow, respectively. For small Prandtl number liquids ( $\operatorname{Pr} \leq 0.01$ ), the perturbation energy mainly comes from the interaction between the perturbation and the basic flow, which suggests that the instability mechanism is hydrodynamic in nature. The interaction between the perturbation and the basic flow in the azimuthal direction becomes negative when a moderate rotation is applied $(\tau=50)$, and the moderate rotation can stabilize the thermocapillary flows for small Prandtl number liquids.

\section{Acknowledgments}

This work has been supported by the National Natural Science Foundation of China (Nos. 11532015 and 11272320).

\section{Conflict of interest}

The authors declare that they have no conflicts of interest to this work.

\section{Appendix A. Supplementary material}

Supplementary data associated with this article can be found, in the online version, at https://doi.org/10.1016/j.ijheatmasstransfer. 2017.09.137.

\section{References}

[1] M.F. Schatz, G.P. Neitzel, Experiments on thermocapillary instabilities, Annu. Rev. Fluid Mech. 33 (1) (2001) 93-127.

[2] J.J. Derby, Y.-I. Kwon, A. Pandy, P. Sonda, A. Yeckel, T. Jung, G. Müller, Developing quantitative, multiscale models for microgravity crystal growth, Ann. NY. Acad. Sci. 1077 (1) (2006) 124-145.

[3] T.A. Campbell, M. Schweizer, P. Dold, A. Cröll, K.W. Benz, Float zone growth and characterization of $\mathrm{Ge}_{1-\mathrm{x}} \mathrm{Si}_{\mathrm{x}}(\mathrm{x} \leq 10$ at\%) single crystals, J. Cryst. Growth 226 (2) (2001) 231-239.

[4] R. Rupp, G. Müller, G. Neumann, Three-dimensional time dependent modeling of the Marangoni convection in zone melting configurations for GaAs, J. Cryst. Growth 97 (1) (1989) 34-41.

[5] H.C. Kuhlmann, H.J. Rath, Hydrodynamic instabilities in cylindrical thermocapillary liquid bridge, J. Fluid Mech. 247 (1993) 247-274.

[6] M. Levenstam, G. Amberg, Hydrodynamical instabilities of thermocapillary flow in a half-zone, J. Fluid Mech. 297 (1995) 357-372.

[7] M. Wanschura, V.M. Shevtsova, H.C. Kuhlmann, H.J. Rath, Convective instability mechanisms in thermocapillary liquid bridges, Phys. Fluids 7 (5) (1995) 912-925.

[8] Q.S. Chen, W.R. Hu, V. Prasad, Effect of liquid bridge volume on the instability in small-Prandtl-number half zone, J. Cryst. Growth 203 (1) (1999) 261-268.

[9] M. Lappa, R. Savino, R. Monti, Three-dimensional numerical simulation of Marangoni instabilities in non-cylindrical liquid bridges in microgravity, Int. J. Heat Mass Transf. 44 (10) (2001) 1983-2003.

[10] M.K. Smith, S.H. Davis, Instabilities of dynamic thermocapillary liquid layers. 1. Convective instabilities, J. Fluid Mech. 132 (1983) 119-144.

[11] Q.S. Chen, W.R. Hu, Influence of liquid bridge volume on instability of floating half zone convection, Int. J. Heat Mass Transf. 41 (6-7) (1998) 825-837.

[12] D. Schwabe, Hydrothermal waves in a liquid bridge with aspect ratio near the Rayleigh limit under microgravity, Phys. Fluids 17 (11) (2005) 112104.

[13] R. Savino, R. Monti, Oscillatory Marangoni convection in cylindrical liquid bridges, Phys. Fluids 8 (11) (1996) 2906-2922.

[14] Q.S. Chen, Y.N. Jiang, Instabilities of vortex rings generated by surface-tension gradients between co-axial disks, Int. Commun. Heat Mass Transf. 39 (10) (2012) 1542-1545.

[15] D. Gottlieb, S.A. Orszag, Numerical Analysis of Spectral Methods: Theory and Applications, SIAM, Philadelphia, 1977. 